\title{
RENÉ VILLARD, BIOGRAPHE DE MAX JACOB
}

Patricia SUSTRAC*

$\mathrm{P}$ our qui s'intéresse à la biographie de Max Jacob, le patronyme des Villard deviendra vite familier. L'enfance de Jacob évoluait dans un Quimper où cette famille puisait une partie de ses racines et y demeure aujourd'hui encore bien connue. Les touristes peuvent toujours acquérir les fameuses cartes postales de Joseph Villard devenu photographe à l'instigation de son frère Jean-Marie, élève de Nadar et de Daguerre, ou celles de Joseph-Marie, son fils, fondateur de la célèbre collection $J O B$. Quant aux gourmands, ils peuvent se régaler des confitures de l'entreprise prospère d'Abel en collectionnant de charmants chromos !

Jacob rencontra d'abord Jean-Marie Villard, professeur de dessin au lycée de Quimper $^{1}$. Le rude enseignant n'appréciait pas les esquisses de son jeune élève ${ }^{2}$. Mais il considérait sûrement d'un œil bienveillant l'amitié entre ses fils et ce jeune homme qui dégustait nonchalamment une boîte de sardines assis sur le mur du vieux cimetière de Ploaré en face de chez lui en attendant qu'on veuille bien l'inviter, ce qui ne manquait jamais d'arriver. De ses deux fils, Abel et René, c'est

\footnotetext{
* Présidente de l'Association des Amis de Max Jacob, Patricia Sustrac travaille à la fois sur des questions biographiques et sur les correspondances jacobiennes. Elle a publié Les lettres de Max Jacob à Roger Toulouse, Troyes : Les Cahiers Bleus, 1992 (en collaboration avec Christine Van Rogger-Andreucci); Lettres à un jeune homme, 1938-1944, Paris : Bartillat, 2009 (lettres à J.-Jacques Mezure) ainsi que plusieurs articles ou notices. Elle prépare la publication des lettres inédites de Max Jacob à Maurice Sachs (1926-1930) ; à Louis Dumoulin (1938-1943) ; à Marcel Métivier (1938-1944) et un essai concernant les mesures de spoliation prises à l'encontre de Max Jacob et de sa famille pendant l'Occupation.
} 
René, le cadet, qui fut le préféré du poète. D'un an seulement plus âgé que Jacob, René aimait rêver, et par-dessus tout versifier. Ce goût de l'écriture qu'il conserva toute sa vie ${ }^{3}$ lui coûta son année de Rhétorique : il redoubla. Sans doute fut-il mécontent, sa famille aussi (elles le sont toujours) mais repiquer lui permit de rencontrer son alter ego Max Jacob. Les amitiés de l'enfance ne s'oublient pas : elles sont « les unions de tempérament, les affinités presque physiques ou astrales, car [elles] ne dépendent pas des circonstances ${ }^{4} \gg$. L'amitié entre Jacob et Villard est née dans l'osmose des complicités sans fards et d'une passion partagée pour la terre natale : « Nous n'avons pas eu la même nourrice, mais nous avons eu la même mère, la Bretagne » écrivait Villard" . "Si l'amitié était une plus profonde connaissance de l'ami, la nôtre n'aurait pas d'égale car, d'être le fruit d'une même terre, d'un même lycée, de mêmes groupes, de mêmes études, après la séparation, n'est-ce pas se connaître plus précisément encore qu'on ne le croit ? $^{6} \gg$ confirmait le poète en 1930 .

En ce début de $\mathrm{Xx}^{\mathrm{e}}$ siècle, les « copains » des deux jeunes gens sont bien connus. Les inséparables sont rejoints par Abel Villard, Olivier Moisan, Hyppolite Piouffle, Joseph-Émile Poirier et les deux frères Bolloré7. On sait aussi de quelle manière ce groupe souda son amitié. Leurs pensées du moment, leurs aspirations et leurs rêves seront consignés, à l'initiative de René, sur un cahier réservé à cet usage dénommé par leurs auteurs Le Cahier des maximes ${ }^{8}$. Ce cahier de deux sous révèle de jeunes collégiens instruits, cultivés et surtout confiants au seuil d'un siècle d'espérances et de progrès qui ne leur avait pas encore appris à gémir. René Villard y reconnaîtra surtout les prémisses du génie de son ami dont il suivait attentivement la carrière'. Sa bibliothèque personnelle, conservée en l'état par Marguerite Floc'hVillard, sa fille aînée, témoigne des lectures approfondies de l'œuvre poétique et romanesque de son condisciple. Ces ouvrages et un matériel documentaire abondant ${ }^{10}$ recueillis par René Villard devaient étayer ses conférences et ses articles ainsi que le projet d'une future biographie ${ }^{11}$ du poète quimpérois.

Ce vaste ensemble a été définitivement rassemblé et classé lors de deux campagnes d'inventaire des archives de Marguerite Floc'h-Villard que j'ai réalisé en 2008 et 2009. Quelques notes éparses avaient été partiellement publiées par l'éditeur des correspondances de Max Jacob à René et Abel Villard ${ }^{12}$. Nous les enrichissons par des documents inédits formant le canevas de deux projets éditoriaux : une conférence [texte 1] et des esquisses de chapitres du projet biographique [textes 2 à 6]. Cet ensemble révèle la réflexion d'un chercheur impliqué dans un travail de synthèse, mobilisé par le souci pédagogique de faire 
connaître une œuvre littéraire et d'en saisir le dessein. Ce matériel est précieux. Il permet d'observer la composition d'une biographie à laquelle le poète n'était pas opposé et d'identifier les ressorts de la construction de la légende jacobienne. Si Villard était, à juste titre, profondément conscient de la dimension religieuse et des tensions contradictoires à l'œuvre dans l'esthétique de Max Jacob, il n'est pas certain toutefois qu'il ait pu échapper, à terme, à une évocation hagiographique tant l'empathie avec le poète était grande. Jacob était et demeura pour Villard «le jongleur de Notre-Dame». Cette référence évidente à la célèbre légende le conduisit dès 1930 à souhaiter l'érection d'une statue "sous le chapiteau gothique d'un pilier de [1'église] de Ploaré, taillée dans le granit de "chez nous". Et les fidèles liront, sous le socle qui soutiendra son image : "Max Jacob, poète et convertisseur, dit le Jongleur de Notre-Dame de Ploaré" "13 ou à composer une ode poétique éponyme en 1936 dédiée à celui « qui tend[ait] ses bras vers le Sauveur $»^{14}$.

C'est en se rendant à une conférence qu'il devait prononcer sur l'œuvre du poète en décembre 1939 que « son cher ami d'enfance, celui qui en fut le plus bel ornement $^{15}$ » s'écroula frappé d'une congestion cérébrale. Il succomba quelques temps après. Inquiet, Jacob sollicita des nouvelles qu'il pressentait très graves auprès de Mme Villard :

Samedi 23 déc. $39^{16}$

St-Benoît- sur- Loire (Loiret)

Madame,

Je suis profondément remué, chagriné douloureusement par la nouvelle que l'on m'apprend de Quimper. Que puis-je croire de ce que m'écrit ma sœur? En quel état est le pauvre cher René? Que lui est-il arrivé exactement? Je serais certainement parti à St Brieuc si j'étais en état de voyager mais ma santé n'est hélas pas brillante. Quelqu'un de votre maison voudra peut-être bien me parler des circonstances de cet atroce accident. Je prie le Seigneur qu'il n'ait pas de conséquences.

Croyez-moi, Madame, avec vous et les vôtres et en union de prières de tout cœur.

Max Jacob 
Cette première lettre doublait un télégramme envoyé le même jour.

\author{
St-Benoît-sur-Loire \\ Loiret \\ le 29 janvier 1940
}

Chère Madame,

Je suis toujours très inquiet de la santé de notre pauvre René. Les nouvelles que j'en ai sont très indirectes et par conséquent très vagues. Serait-ce trop demander à vous, Madame si occupée et préoccupée, de me mettre quelques lignes sur une carte postale. Quelqu'un de votre entourage voudra peut-être bien s'en charger. Je prie chaque jour pour René et sa famille.

Croyez, Madame, à ma respectueuse compassion et veuillez agréer mes hommages.

Max Jacob

Mme Villard garda le silence : toutes les archives de René Villard se refermèrent et restèrent muettes. Elles s'offrent aujourd'hui dans leur intégralité à tous les chercheurs jacobiens et à tous les historiens de la Bretagne par la création d'un « fonds René Villard » à la Médiathèque des Ursulines de Quimper grâce à la générosité de sa fille Marguerite et le truchement de l'association des Amis de Max Jacob. «Que tu as raison quand tu dis que notre amitié est de celles qui durent jusqu'à la mort » écrivait Jacob à Villard en $1933^{17}$. La constitution de ce fonds corollaire à celui du poète rend désormais éternel ce lien fraternel de l'enfance.

Note d'édition :

La publication des manuscrits n'a pas présenté de difficulté majeure de déchiffrement. L'écriture de René Villard a permis une transcription presque littérale du texte à l'exception de quelques termes hâtivement tracés ou d'abréviations insolites. Marguerite Floc'h-Villard a décrypté aisément les volutes élégantes de l'écriture paternelle. Francis Deguilly est venu à bout de termes récalcitrants. Que tous deux soient chaleureusement remerciés. Toutefois, rapporter dans le détail les multiples abréviations et ellipses dues à l'écriture hâtive de Villard n'apporterait aucun intérêt au lecteur. C'est pourquoi les mots ont été retranscrits dans leur orthographe habituelle en restituant une ponctuation 
conventionnelle ainsi qu'une orthographe et une grammaire adéquates. Les conventions typographiques ont été appliquées aux titres des ouvrages que René Villard omet de souligner ou de mettre entre guillemets. Les ajouts nombreux placés dans les marges des feuillets ont été réintroduits dans la logique du texte. Les mots soulignés sont transcrits en italique. Les ajouts facilitant la compréhension sont placés entre crochets [], les variantes du manuscrit avec le texte 1 qui a été publié sont placées entre accolades $\{$.

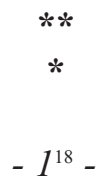

Sur le disque éclatant de l'Odet élargi

J'aimais apercevoir entre les doigts des arbres

Les joues du grand voilier dorées par le soleil

Tandis que sous nos pieds s'élançant des broussailles

Les trois-mâts fins et lourds faisaient songer à Dieu. J'écris nos deux clochers en lettres majuscules... ${ }^{19}$

\{Tout Max Jacob se trouve condensé dans ses lignes et tout Quimper... Quand on les a lues, on saisit l'amour ardent que Max garde à sa ville natale et sa sensibilité de poète à la Bretagne s'y révèle tout entière\}.

Max Jacob malgré l'éloignement, malgré les épreuves a gardé pour sa ville natale : Quimper, un vrai culte. Quand il prononce ce nom aux deux syllabes sonores comme une chute d'eau, il a sans doute la ferveur d'un Italien parlant de Florence. Mais n'a-t-on pas dit que Quimper était une Florence bretonne ? Contrairement à beaucoup d'écrivains qui ont conservé de leur collège un déplorable souvenir, Max parle toujours de son vieux lycée avec attendrissement. C'est une vieille maison où il a laissé un peu de son cœur. Il est vrai qu'il y fut un élève brillant. N'a-t-il pas remporté en Philosophie un accessit ? Cet accessit lui ouvrait les portes des lycées de Paris. Il dédaigna de profiter de cet avantage. C'était un oiseau sauvage qui avait horreur de la cage, je ne le vois pas vivant la vie d'internat dans ces grandes casernes universitaires de la capitale. Sans doute, il fut choyé de la plupart de ses professeurs, tous l'aimaient : «J'ai un souvenir attendri, m'écrit-il, du professeur de Préparatoire. Il aimait particulièrement Louis Sablé et moi : il nous prenait par la main et nous faisait sauter autour de la cour en chantant un air que j'ai retenu : “ Ma sœur est heureuse/ c'est la mère Bidard/ moi j'suis blanchisseuse/ dans la rue Mouffetard “. Je n'ai plus jamais entendu cette chanson depuis $»^{20}$. Mais, ne trouvez-vous pas, cher 
lecteur, qu'elle n'étonnerait pas si on la lisait dans Cinématoma ou dans Les Pénitents en maillots roses? \{Elle a toute la cadence, la naïveté, le charme roturier de la plupart des « chansons » de Max Jacob\}. \{Comment ne pas aimer un maître, serait-il effroyablement louche, comme l'était ce professeur quand il [1']a fait danser au son d'une chanson enfantine ?] M. Riou ${ }^{21}$ ne fut pas une exception. Max fut un élève aimé de ses maîtres. Il avait une manière à lui de les séduire. L'abbé Hamelin, son professeur de $4^{\mathrm{e}}$ avait un faible aussi pour Max bien qu'il fût [juif, poète et athée] ${ }^{22}$, prêtre et bien que Max fût hors de l'Église. Avait-il pressenti qu'un jour il y entrerait comme converti ? Qui sait? \{Je n'ai pas été le condisciple de Max dans la férule, je dis bien la férule du père Hamelin. Nous étions des «copains ». Nous passions ensemble les après-midi des jeudis et des dimanches dans la maison hospitalière du quai de l'Odet qui sentait le vieux bois et les étoffes fanées\}. Mais c'est beaucoup plus tard en Rhétorique que je suis devenu le condisciple de Max, sous la bienveillante direction de M. Surer, si profondément convaincu, intelligent et pondéré. Par un concours de circonstance assez curieux, la classe de Rhétorique à Quimper en 1893 comptait un nombre appréciable d'apprentis hommes de lettres. Le nom de Raoul Bolloré reste indissolublement attaché à celui de Max adolescent. Lui-même n'a t-il pas écrit de Bolloré : « Ne vous souvenez-vous pas de ce poète de 13 ans qui avait du génie ? ». Max avait deviné que ce jeune homme blond aux gros yeux de myope aurait pu en effet devenir un de nos plus grands poètes, s'il n'était mort à 20 ans, d'une mort volontaire. Max avait une affection profonde pour Raoul Bolloré. Mais il existait entre ces deux jeunes gens destinés à se faire un nom dans les lettres une rivalité qui vaut d'être contée. Mais laissons les rivaux raconter eux-mêmes leurs joutes littéraires : « Hier soir, écrit Raoul \{à son père\}, à la classe d'histoire, M. Wattecamps rendait compte à Jacob et à moi d'un devoir que seuls dans la classe nous avions fait et qui consistait dans un portrait de Chilpéric. Naturellement rivalité entre Max Jacob. Le devoir de ce dernier (il en était assez vexé) a été mis en opposition avec le mien (en faveur du mien). De plus, sa dernière dissertation a fait un four immense (ce qui le mit en fureur folle contre moi)- notez bien que c'est l'opinion d'un rival doué d'une puissante imagination ${ }^{23}$. Et en ce moment nous luttons dur et ferme à grands coups de plume et d'imagination, jusqu'ici la victoire est pour moi. Mais un vieux proverbe dit que la Roche Tarpéienne est près du Capitole ». Cette sage réflexion prouve assez nettement que si la victoire était cette fois du côté de Bolloré, il considérait Max comme un rival redoutable. La joute «Bolloréo-Jacobine » comme Bolloré dénommait cette émulation dura toute l'année. Elle fut interrompue en Philosophie. Bolloré rencontra la Roche Tarpéïenne au Baccalauréat bien qu'il fût prix d'honneur de français. Max distança d'une année son adversaire en regrettant d'ailleurs de ne plus avoir à se mesurer avec son grand ami et rival le poète Raoul Bolloré. 
Nous avons conté dans un article qui a paru au Correspondant la joute extra scolaire qui mit en présence les deux rivaux de la classe de Rhétorique. Résumonslà pour ceux qui n'ont pas eu connaissance de cet article. Nous étions tous en Rhétorique cette année 1893-1894 plus ou moins des apprentis littérateurs. Raoul Bolloré, Max, le poète J. É[mile] Poirier aujourd'hui rédacteur au Petit Journal (j'en étais aussi) eurent l'idée de fonder un journal que nous appelions je ne sais pourquoi La Cigogne. Malheureusement l'administration s'en mêla, confisqua le journal et firent subir aux jeunes rédacteurs un interrogatoire sévère comme on fait subir aujourd'hui aux cagoulards). Aujourd'hui les professeurs pour occuper les "loisirs » persuadent les élèves des lycées de fonder des revues scolaires. Au lycée de Quimper en 1893, les élèves journalistes étaient suspects et on leur infligeait des consignes ${ }^{24}$. La Cigogne persécuté reparut sous un autre titre beaucoup moins littéraire : Le Club de l'Universel. Max Jacob n'y collabora pas ou très peu. En revanche il collabora à une autre ouvre qui ne quitta jamais la salle de rédaction de la rue de Douarnenez car j'en avais la garde. Chaque fois que je recevais un ami, il était tenu d'y écrire une maxime (de là le nom de ce cahier), une poésie, un portrait. Si le génie était premier, c'est à cette création que je dois de posséder les premiers poèmes en prose de M. Jacob. Il s'essayait déjà dans l'ironie à froid et s'exerçait dans ce genre mi-sérieux, mi-fantaisie, qui a fait la fortune de ses livres.

Max y consignait des maximes à La Rochefoucauld : "On commence d'abord par louer mille qualités d'un écrivain, on les lui ôte ensuite une à une ». À cette question : "Quelle est la plus jolie femme de Quimper? ", Max répondait : " C'est la plus laide, je l'aime ». Ou bien il écrivait : "Je me défie des vertus exagérées. Plus grand est le vice, plus épais doit être le voile qui le recouvre $"{ }^{25}$. Sous l'ironiste et le jongleur, nous découvrons déjà le " moraliste ». Je regrette de borner là ces citations. Mais il n'éclatait pas aux yeux que ce cahier de maximes, ce modeste cahier de deux sous, a fourni le cadre où le génie naissant de Max Jacob a commencé d'évoluer et de s'épanouir. N'aurait-il que ce mérite, c'en fut un. L'institution du cahier fut la source d'où devait jaillir Le Laboratoire central, Le Cinématoma et surtout le Cornet à dés dont Georges Gabory a pu dire qu'il était le "plus riche écrin de sensations \{curieuses\}, d'images neuves, d'impressions inconnues, une des plus singulières et des plus belles expression poétiques $\left\{\right.$ lyriques\} de ce temps $\|^{26}$.

Il n'est donc pas téméraire de prétendre que Max doit beaucoup au milieu universitaire où il vécut de 1800 à 1896. Les maîtres : Surer en Rhétorique, Dugas en Philosophie, le poète Abbé Hamelin en $4^{e}$ ont eu sur son esprit et sur son talent une grosse influence, mais Max doit aussi beaucoup à Raoul Bolloré, à Olivier Moisan, morts depuis, et à quelques autres qui vivent encore comme J.-É. Poirier qui ont fait dans les lettres une honorable carrière. 


\section{Max Jacob}

Hier dimanche je vais à la messe de 7 heures dans l'intention de communier. Je me confesse au curé puis je vais m'installer face au pilier devant la Sainte Table. Avant la communion, j'entends un pas ouaté : c'est Max qui arrive chaussé d'espadrilles blanches malpropres. Il a son petit veston violet qui n'est pas à sa taille et son large pantalon de coton forme charleston qui fait cloche sur ses longs pieds. Son cou est enveloppé d'un long foulard blanc et bleu qui fait bourrelet : il cache l'absence de faux col. Il a dû se confesser la veille car à l'heure de la communion, il s'avance à la Sainte Table et reçoit l'hostie puis il se rend à sa place et il fait son acte d'adoration, une jambe tendue, l'autre repliée, le pied droit appuyé sur le prie-Dieu dans une curieuse attitude d'enfant mal élevé.

Ce matin dans l'église que remplit la parole des simples, jeunes filles en jupes courtes et en bonnet qui forment cercle autour de lui : vraiment il fait contraste (avec sa grosse figure tannée et sa tête ronde chauve sur le sommet et rasée de près comme un moine). Il fait contraste avec cette assemblée campagnarde que son attitude de bohème pieuse ne paraît pas scandaliser. Une paix religieuse remplit le vaisseau silencieux ; une pauvre lumière - car le ciel est gris - traverse la maîtresse vitre dont les couleurs rutilantes essayent d'illuminer la pénombre. La veilleuse se balance au plafond voûté et les lueurs rayonnantes vacillent sur les pieds des grands chandeliers de cuivre de l'autel, comme la lumière d'un phare à éclipse. Le prêtre à l'autel murmure à voix confuse les paroles liturgiques comme un homme innocent qui prononcerait des paroles incohérentes et le rectangle vert de sa chasuble se promène de l'épître à l'évangile. Messes matinales dans les petites églises campagnardes ! Combien de poètes déjà ont dit votre charme primitif...

Après la messe je m'arrête sous le porche verdi, sous la statue peinte de Dieu le Père qui porte un globe dans sa main. Max me rejoint dans cette pénombre verte sous les niches vides ${ }^{27}$; sous les [mots manquants] des voûtes patinées par les siècles. «Tiens, toi, là, René. Je t'avais aperçu dans l'église mais je ne t'avais rien dit à cause de la Sainteté des lieux. » Max m'offre de prendre le café dans une petite auberge. Nous nous asseyons comme les gens du pays devant une petite table de bois vernie dans une salle décorée de bouteilles à étiquette chromos. Là, nous 
causons longuement. Je lui parle de sa poésie «Le chien noir » qu'il m’a offerte la veille avec un dessin et je lui dis que mon frère le docteur a trouvé du style au dessin et de la valeur au poème ${ }^{28}$.

Il tourne vers moi ses yeux verts et clignant tantôt l'œil droit, tantôt l'œil gauche, passant d'un geste qui lui est familier les doigts de la main sur son nez en caoutchouc : «Tu sais, je m'en fous, je m'en fous, ce qui veut dire, qu'on dise du bien de moi ou du mal, je m'en fous ». Puis il me parle de l'Argus de la Presse qu'il a cessé de recevoir, parce que chaque jour ces coupures lui apportaient des sottises : " "Max Jacob est un idiot, un fumiste...". Ma vie est une vie de martyr. Je suis très mal avec Gallimard, mon éditeur... ».

« Et pourtant il t'imprime »

« Oui, parce que j'ai un contrat»

« Je suis très mal avec $\mathrm{X}$ et $\mathrm{Y}$... Tu ne sais pas ce que c'est, les gens me détestent » « Mais alors comment se fait-il qu'on trouve partout ta prose ou tes vers?»

«Je n'ai plus qu'une ambition : gagner ma pauvre vie. Pour ce que je fais, je trouve à le placer. Je ne propose rien. J'écris comme ça chaque jour, ce que j'ai à dire, sans m'occuper de rien. Quand on me demande quelque chose, je dis : “ Tiens, voilà "et ainsi il [ne] me reste rien. On me paye ou on ne me paye pas. Car les grandes revues ne veulent pas de moi, je n'écris que dans les petites revues »

«Oui, Philosophies ${ }^{29} »$

«C'est cela Philosophies et d'autres du même genre. Mais je ne m'occupe plus de ce qu'on dit de moi. Mon nom est "fait", c'est certain, ça je l'accorde, tant mieux pour [moi] et je dessine, et, je dessine énormément. Songe donc René, j’ai bien publié trente volumes, oui, trente volumes, ce n'est pas mal. Je ne suis pas marié, je n'ai pas pu me marier- je ne pouvais avoir une femme et mener la vie que j'ai menée. Du reste l'homme de lettres ne peut se marier, il faut choisir. »

Je lui demande s'il aurait encore quelques volumes de La Côte qui a été récemment publiée avec des gouaches. «Il ne m'en reste plus. Tu sais, cela vaut très cher. Je les ai vendues toutes. Il faut bien vivre. C'est ce qui m'a permis de faire ce voyage en Bretagne. » « Et ici comment vis-tu ?»; « Je me lève à 6 heures tous les matins. Il le faut bien avec tout ce que $\mathrm{j}$ 'ai à faire. »

Puis il parle du jeune P[ierre] C[olle] qui se dissipe, qui va tous les soirs au bal.

« J'ai une grosse responsabilité ; voilà un enfant que j'aime beaucoup, mais il se perd, il est sur une terrible voie, bien que très pieux. »

«Tu n'en es pas responsable. » 
« Mais si, mais si, tu sais bien que nous portons les péchés les uns des autres. Tous nous portons les péchés des uns des autres. Et moi, j'en ai beaucoup déjà à me faire pardonner. Il faut agir. Il ne suffit [pas] de dire « Mon Dieu ! Mon Dieu » : il faut aller à la communion ; à quoi cela servirait de toujours implorer notre Père si nous ne prenions pas le remède, le remède c'est l'hostie. C'est l'hostie qui opère. Autrement, c'est l'enfer, l'enfer. »

Et longuement nous bavardons de mille choses. Je quitte Max. Il s'en retourne avec son gros paroissien sous le bras, un missel imprimé en Belgique. « Il faut que j'aille faire mon Chemin de Croix et dire les psaumes de la pénitence car tous les jours je fais mon Chemin de Croix et je suis les psaumes de la pénitence. Il le faut bien... » Je reconduis Max jusqu'au porche sous la pluie ; lui signalant qu'il doit éviter les flaques d'eau où trempent ses pieds chaussés d'espadrilles. Il serre autour de son cou qui n'a pas de faux cols son cache-nez blanc et bleu qui lui fait comme un bourrelet autour du cou.
«Au revoir Max... »
«Au revoir René... »
9 août $1927^{30}$

$$
-3-
$$

\section{Max Jacob, 14 août 1928}

Max est installé ici chez M. le Mony, l'entrepreneur. Petite chambre claire dont la fenêtre s'ouvre sur Douarnenez, l'horizon et la mer. Une grande planche sur deux tréteaux lui sert de table et il y accumule ses livres (Bible de Crampon), ses pastels, ses godets à gouache ; tout un attirail de peintre écrivain. Il traîne pour moi au milieu de la pièce un vieux fauteuil au rouge passé et je m’y installe pour regarder les murs blancs auxquels pendent des images pieuses : un saint Joseph couvert de bure portant un petit Jésus en chemise ; une sainte famille avec des figures crépues et des auréoles d'un or très pur. Une image de Notre-Dame de Lourdes les mains jointes et les pieds couverts de roses. Voilà de quoi s'inspire l'auteur de La Défense de Tartufe. Max est en chemise de zéphyr au col largement dégarni, laissant voir les poils blancs de sa poitrine velue ; avec ses joues grasses, sa cigarette, sa tête rubiconde de Frère des Entommeurs, il ressemble à Balzac, un Balzac très torve, trapu, ventru, dont le pantalon gris lui serre le ventre comme une sous-ventrière équestre... Il suce l'extrémité d'une cigarette, dont ne s'exhale aucune fumée ; il ne tient pas en place, il va, il vient, culbute Pierre Colle (car 
P. Colle est là sur le lit) qui est défait ; ou bien Pierre Colle s'empare des bras gras de Max et esquisse avec lui un charleston. Très comique vraiment ! Très Montmartre, bien que nous soyons à huit cents kilomètres de la Butte. J'interroge Max qui répond à mes questions précises :

«Es-tu marié ?»

« René, non, c'est faux, tu l'aurais su, rien de grave ne se passe dans ma vie sans que tu le saches, je t'aurais au moins envoyé un mot ; et si je meurs Pierre Colle ici présent se chargera de t'annoncer la nouvelle. »

!!!!!

Max s'interrompt pour s'emparer d'un chiffon blanchâtre fortement teinté de pastel vert. Est-ce un mouchoir? Est-ce un chiffon d'atelier? Je ne m'en suis pas informé pour ménager la susceptibilité de Max et l'interrogatoire continue.

«Est-il vrai que tu aies quitté St-Benoît?»

« Nullement, nullement, je suis toujours à St-Benoît, seulement je ne veux pas m'astreindre à demeurer tout un hiver à la basilique sans en bouger. »'

Pierre Colle : « Max a des affaires à régler à Paris ; il faut qu'il s'occupe de ses rééditions, de ses expositions - on prépare une quinzaine Max Jacob à $L a$ Renaissance. Je dois faire un article pour La Renaissance sur Max Jacob peintre. »

Je me lève pour voir les gouaches de Max. Il y en a une quinzaine. Je les examine une à une. Voici Locronan avec sa tour qui est bien dans l'atmosphère; la couleur n'est pas fausse, c'est bien le jus de chique de la vieille pierre et les maisons qui se groupent autour. Son dessin est, ma foi, avec un respect surprenant de la perspective. Le triomphe de Max, ce sont les blancs. Ses façades brillent comme de l'argent et ses toits c'est encore de l'argent bleui. Évidement cela a un certain charme. Ce sont surtout les premiers plans qui sont maladroits. C'est barbouillé de gris ou rose et l'on ne sait pas si c'est du trèfle, du blé ou du foin. On ne sait pas non plus ce que c'est que ces plantes du premier plan qui sont d'une naïveté d'enluminure du XV $V^{\mathrm{e}}$ siècle. L'ensemble se tient.

Pourtant Max me montre une marine qu'on dirait peinte par un quartiermaître en retraite et où quelques bateaux à voiles blanches évoluent sur une mer de lait : «Celle-ci, mon cher a été refusée, refusée, tu entends, le marchand me l'a renvoyée... Et pourtant c'est l'exactitude même. » Max n'en revient pas de cet échec. Mais Max n'en n'est [pas] désespéré. J'examine un certain nombre de gouaches : un Jésus guérissant un aveugle avec un énorme bras droit qui lui pèse, une robe pourpre. Ce qu'il y a de réussi c'est le paysage, vraiment palestinien avec ses petites routes en lacets, dans la plaine plantée d'oliviers. Au fond le lac 
de Tibériade d'un bleu sale et la ligne ténue des monts. Je vois aussi un nu de Philippe $^{31}$ et de nombreux dessins du même éphèbe !

Puis nous parlons de livres. De prix littéraires. Je lui parle de Louis Guilloux qui vient d'avoir le prix Blumenthal ${ }^{32}$ et il me dit avec une nuance de pitié et une chaleur un peu indignée : «Tu sais, ne te laisse pas prendre, ces prix n'ont aucune valeur. C'est une charité. C'est une charité qu'on a faite à Guilloux, à Pierre Gueguen qui avait sa femme très malade et les soins lui coûtaient $\operatorname{chers}^{33} \ldots$ Et Marcel Sauvage qui n'a fait que me voler ma manière. C'est un plagiaire et beaucoup d'autres qui n'ont aucun talent. » Y aurait-il un peu de dépit au fond du cœur de Max qui fait partie de nombreux jurys littéraires et qui n'en a eu aucun ${ }^{34}$ ?

J'examine quelques-uns des livres de Max qui s'empilent sur la cheminée : je lis les titres : Le Cabinet noir, lettres sans commentaires; le Soniou Breiz-Izel de Le Braz et Luzel (il lui fallait de tels livres pour écrire La Côte) ; et ceux des livres nouvellement parus d'auteurs encore inconnus qui feront parler beaucoup d'eux demain. Max me prête son Cornet à dés dont il révise le texte en vue d'une prochaine édition et il rédige pour le directeur du Mail, la revue d'Orléans, qui a parlé de lui et que je désire posséder. "Tu es un honnête homme », me dit-il en prenant son édition unique du Cornet à dés.

$$
-4-
$$

\section{Max Jacob, 15 août $1928^{35}$}

Aujourd'hui, fête de l'Assomption, je communie à la messe du matin. Je m'étonne de ne pas voir Max à la communion de 7 heures du matin. Mais à la fin de la messe j'aperçois sa silhouette massive, cheveux drus et blancs autour de son crâne chauve, petit veston ballant, sandales blanches. Il jette un coup d'œil autour de lui, puis s'agenouille à la Sainte Table, le corps tout penché dans une attitude de recueillement et d'humilité, il attend la Sainte Hostie. À la sortie, je lui serre la main dans l'église et nous allons ensemble boire un café au lait dans la buvette de Le Roux, en face de l'église ; nous causons. Il me parle de son besoin de communier. Sa santé est mauvaise. Le matin, il se lève malade, il se traîne à l'église, mais dès qu'il a communié, il reprend des forces. « Je serais malade si je ne communiais tous les jours. Et dire qu'il y a des gens qui prétendent que c'est de l'autosuggestion. » 
Puis, nous parlons de ses livres, de son œuvre. Il est mélancolique, désabusé. « La gloire ! Je m'en fous, maintenant que je l'ai, je suis comme cet homme de Lorient qui disait : “ Quand j'avais des dents, j[e n']avais [pas] de bécasse, maintenant j'ai de la bécasse, mais je n'ai plus de dents “. Je suis comme lui, j'aurais voulu la gloire à 20 ans, elle est venue trop tard, maintenant je ne puis plus en profiter. Je m'en fous, ce que je veux c'est gagner ma vie, avoir un peu d'argent $»^{36}$. Je lui parle de son album de dessins qui est épuisé, de son Saint Matorel qui n'a pas été réimprimé, de tous ces ouvrages qui font prime et qui sont maintenant épuisés.

Puis il me parle de ses frères : Maurice, Gaston. Le premier est maintenant un vieillard, il est marié et sans enfants, le plus jeune aussi est sans enfants. Gaston n'est pas marié. Et je lui dis : « Mon pauvre vieux, ton nom se perdra ». " Oui, et je le regrette » ajoute-t-il mélancoliquement. Peut-être est-ce pour qu'il ne meure pas qu'il a pensé à se marier ${ }^{37}$.

Comme nous descendons la rue de Ploaré, il me parle de la reconnaissance de son élève Philippe Lavastine que ses parents lui ont confié pour tâcher de le convertir. Le voilà parti pour Belle-Île pour voir sa p.... ( « Tu sais ce que c'est qu'une $\mathrm{p} . . .$. ? » me demandait-il avant hier). «Quand on pense que ces gens-là ne m'ont pas envoyé un mot, quand j'ai obtenu de Philippe qu'il se confesse et communie cette année. Ils n'ont aucune reconnaissance. Il a communié, c'est bien ; rien de plus... $\gg^{38}$. Max continue son apostolat.

\section{Max Jacob}

29 août [19]28- Hier je vois Max chez J[ean] Colle. Je le trouve couché sur un lit avec un énorme édredon sur le corps. "Mon cher, ça va mal. J'ai mal à l'estomac et l'estomac détraqué ». Max souffre aussi du foie. Il est allé voir le docteur Darney qui prétend que ces malaises doivent être attribués au pain de Douarnenez. Max n'a pas mangé de viande pendant deux ans, il ne s'est pas porté mieux pour cela. Il se lève et vient causer sous la fenêtre. Philippe qui est là feuillette un livre de poèmes de X édité par la N. R. F. dédié à Max Jacob. Philippe lit à haute voix des vers amorphes.

"Je retrouve ta manière, mon cher Max",

"Rien d'étonnant, ils me copient tous » et il lit lui-même ajoutant : "Il y en a ainsi des kilomètres, l'auteur est un brillant élève de l'École des Mines. » 
On parle d'éditeurs. Max nous conte l'histoire d'un M. X qui s'est présenté chez Kra au nom de Max en lui disant qu'il avait besoin de 14000 francs pour écrire un roman. Kra lui donne les 14000 francs et il part je ne sais où avec un ami faire une bombe carabinée avec les 14 000. Il revient chez Kra et lui dit : " Mon roman n'est pas encore terminé, donnez-moi encore 14000 francs pour le finir ». Kra l'envoie promener et poursuit l'escroc, mais il n'est pas remboursé. Pour couvrir ses pertes, il vend Les Pénitents en maillots roses et n'en donne pas un sou à Max.

«Alors il est inutile que je me recommande de toi à Kra »

«Absolument inutile»

«Quel est donc actuellement ton éditeur?»

«C'est Gallimard - malheureusement ! »

« Pourquoi malheureusement?»

« Je suis à lui pour la vie, il n’y a rien à faire et je lui dois 12000 francs ».

Puis on parle de Laennec et d'une biographie de Saint-James. Pierre Colle me conseille d'écrire ce livre, Max approuve : «On pourrait donner ça au Roseau d'Or ». Il est alors question d'un livre de Joseph Delteil sur Max : La vie de St Max $J a c o b^{39}$. Et Pierre Colle ajoute : «Il est piqué ! » Max appuie. Max me montre ensuite deux gouaches dont je dis de l'une : "C'est une vue biblique » - « Non, c'est le Midi, une pure vue de l'esprit ». Dans un paysage doré une ville de lumière se dresse avec ses toits rouges, vision lumineuse et juste.

Mais sa vue de Bénodet, dans une harmonie de vert et de blanc, est encore supérieure. La mer chatoie, le ciel a des tons de gorge de pigeon et le paysage de côte d'une simplicité étrange de lignes a de la couleur. L'ensemble chante à l'œil. C'est de la peinture subjective évidemment. Mais la formule se soutient. C'est vraiment d'un artiste, quoique la touche soit maladroite. «C'est à Pierre Colle, il se charge de vendre ça. Dis bien autour de toi que ces gouaches montent de prix ; c'était 600 francs l'année dernière, maintenant on se les dispute à 2000 ! » L'an dernier Max disait 5000 ! Il est quelque peu méridional ! Je prends congé après avoir jeté un coup d'œil sur les toiles de J[ean] Colle, sur un bois de Guillivic, un bénitier breton.

Max me conte qu'il est allé à Rome, mais il n'est pas allé voir le Pape. Il est allé à Rome à l'occasion du Jubilé : « Tous mes péchés m’ont été remis ». Philippe : « Et ceux que tu commettras dans l'avenir ! », Max : « Ah ! Non ! C'est déjà beaucoup ! ${ }^{40}$. 


\section{Souvenirs de Max Jacob, septembre 1933}

Ma fille me rapporte une conversation avec M. Kerhuel ${ }^{41}$. Kerhuel s'arrête à la devanture de Le Bras (il y a un La Côte de Max Jacob) et entre. " Combien le volume ? »-«5 francs »-« Je vous l'achète ».

Max entre sur ces entrefaites : « Justement j'étais en train d'acheter ton livre ! », « Eh bien mon cher tu es mon premier souscripteur », « Ah ! s'il en est ainsi tu vas me mettre une dédicace ». Et Max d'écrire une dédicace comme il sait les écrire ${ }^{42}$.

Kerhuel raconte aussi à ma fille qu'à l'âge de 40 ans (1916) Max fit sa première communion. Il voulut la faire comme les petits garçons, acheta un brassard blanc, un gros cierge et traversa ainsi Paris.

\section{NOTES}

${ }^{1}$ « Le père Villard (...) était un grand artiste ; on en avait fait un pauvre professeur » (JACOB Max, préface, catalogue de l'exposition « Gouaches et dessins par Max Jacob », Paris, Galerie BerheimJeune, 1920, ex. non paginé).

${ }^{2}$ «Petit sot! Drôle ! Retournez la feuille ! Barbouilleur » (Ibid.).

${ }^{3}$ Villard fut le correspondant de l'Illustration, Ouest-Éclair, La Dépêche de Brest. Il publia aussi des ouvrages poétiques : Poèmes de guerre, 1916 (compte d'auteur) et De l'Aube au Crépuscule (Paris, éd. Messein, 1939) préface de M. Jacob.

${ }^{4}$ Lettre inédite de Max Jacob à Marcel Métivier, 28 août 1939.

${ }^{5}$ VILLARD René, « Histoire d'une classe du lycée de Quimper », Le Correspondant, 10 janvier 1930, p. 79.

${ }^{6}$ Dédicace de Max Jacob du 4 septembre 1930 sur l'exemplaire personnel du Laboratoire central de René Villard.

${ }^{7}$ Raoul Bolloré se suicide le 14 mars 1895 à 20 ans. Son frère aîné se suicide en 1896. Yannick Pelletier évoque deux tapuscrits de Villard consacrés à Bolloré (JACOB Max, Lettres à René Villard suivies du Cahier des Maximes, Mortemart, Rougerie, 1978, ouvrages cités sans mention de date ni d'imprimerie, p. 42).

${ }^{8}$ JACOB Max, Ibid., p. 99 à 119. L'édition ne publie que les réponses de Jacob. Le Cahier des maximes, acquis par la Médiathèque des Ursulines de Quimper en 2008, reste à publier dans son intégralité.

${ }^{9}$ Outre l'article paru en 1930 dans Le Correspondant (op. cit.), René Villard a publié : « Le Chevalier Max Jacob », La Dépêche de Brest, 8 août 1933 ; « Max Jacob, collégien », Le Goéland (1ªr avril 1938). 


\section{René Villard, biographe de Max Jacob}

${ }^{10}$ Important lot de feuillets relatifs à des notes de lecture. La Défense de Tartufe (quatre feuillets) : nombreux passages soulignés, recopiés ou commentés. Au chapitre Journal de la conversion, Villard note : « Max s'analyse, introspection. Il dissèque son orgueil. Il a encore de l'esprit. Véritable confession mais extrêmement intelligente et subtile. Le sentiment n'existe plus, c'est une pensée absolument libre, lucide, d'une clairvoyance de chirurgien qui coupe, taille ». Notes de lectures, commentaires et poèmes recopiés extraits du Cornet à dés et Le Roi de Béotie. L'ensemble, daté du 16 novembre 1939, est le canevas de l'ultime conférence. Villard écrit : « Je n'irai pas à adopter comme mienne la déclaration de Mac Orlan que l'œuvre de Max Jacob est une des plus puissantes parmi celles qui constituent aujourd'hui la littérature essentielle de l'Europe. Mais il est certain d'autre part que le nom de notre compatriote quimpérois est prononcé avec vénération dans l'univers, plus peut-être à l'étranger qu'en France par bien des jeunes écrivains d'aujourd'hui. D'où vient cette notoriété de Max Jacob ? Il me semble qu'elle n'est pas tellement due au mérite de l'œuvre même de Jacob qu'à la personnalité de cet écrivain. Le plus chatoyant poème de Max Jacob c'est sa vie et ses écrits ne sont attirants que dans la mesure où ils en sont le reflet ». Notes éparses concernant La Côte ; article de Louis Gonzague Frick « M. Max Jacob et son Cornet à dés » recopié de la revue Sic (n 24, décembre 1917, 2 année ; SIC, Paris, éd. Jean- Michel Place, 1980, p. 178). Extraits recopiés du compte rendu de Louis Aragon à propos des Mamelles de Tirésias (séance du 24 juin 1917) paru dans $S I C \mathrm{n}^{\circ} 27$, mars 1918 (op. cit. p. 206). Notes à propos d'un compte rendu de l'exposition de peintures de Jacob à la galerie de T. Briant et un feuillet non daté intitulé « pessimisme de Max » reprenant les aphorismes de Jacob contenu dans Le cahiers des maximes (XX, XXII, XXXII, XXXIV) voir JACOB Max, Lettres à René Villard, op. cit., p. 99 à 119.

${ }^{11}$ Le canevas de la biographie est le suivant : Max Jacob de Quimper : années de collège ; le pays de bohème (Carco), le poète montmartrois des humbles et des bêtes, naissance du cubisme ; la conversion (Défense de Tartufe), le pénitent en maillot rose, l'âme mystique et tragique de Max Jacob ; Saint-Benoît-sur-Loire ; évasions (conférencier) ; l'acrobate ; le philosophe ; Morven le Gaélique; le sorcier (astrologue), Max Jacob le mage ; l'ennemi des lois ; le paria/venin (poète maudit)/ critiques, moqueries, etc., Max Jacob poète burlesque.

${ }^{12}$ JACOB Max, Lettres à René Villard, op. cit. et Lettres à René Villard II, même éditeur, coll. Poésie Présente $n^{\circ} 41,1982$.

${ }^{13}$ VILLARD René, « Histoire d'une classe du lycée de Quimper », op. cit., p. 97.

${ }^{14}$ VILLARD René, « Le Jongleur de Notre Dame », Le Journal des étudiants de Rennes, 1936 [De l'Aube au crépuscule, op. cit. ; Aguedal, n² 2, 1939 ; Aguedal, 1944]. À propos de cette légende voir la nouvelle éponyme dans L'Étui de nacre d'Anatole France.

${ }^{15}$ Dédicace de Jacob sur l'exemplaire personnel de René Villard des Pénitents en maillots roses (ex. $\mathrm{n}^{\circ} 711$ sur Vélin) : « Cher ami d'enfance/ Chaque poème de ton humble ami est le reflet de sa jeunesse et tu retrouveras entre les lignes blanches des fantômes qui nous sont également chers./ Mes deux mains cordiales. Max Jacob ».

${ }^{16}$ Nous remercions les ayants droit de Max Jacob et Marguerite Floch' Villard d'avoir autorisé la publication de ces lettres inédites.

${ }^{17}$ JACOB Max, Lettres à René Villard, op. cit, p. 43.

${ }^{18}$ Ce texte est le canevas d'une conférence reprenant de larges extraits de l'article publié dans Le Goéland du $1^{\mathrm{er}}$ avril 1938 (op. cit.). Les variantes sont entre accolades.

${ }^{19}$ Villard cite cinq vers du poème «Quimper » extrait de Le Laboratoire central (éd. du Sans Pareil, 1921 ; Gallimard, 1980, p. 57). L'article du Goéland ne reprend pas cet extrait.

${ }^{20}$ Lettre du 24 novembre 1936 (JACOB Max, Lettres à René Villard, op. cit. , p. 65).

${ }^{21}$ Professeur de la classe préparatoire du lycée de Quimper.

${ }^{22}$ Barré sur le ms, non publié dans l'article. 
${ }^{23}$ Barré sur le ms. Anecdote reprise avec variantes (JACOB Max, Lettres à René Villard, op. cit., p. 79).

${ }^{24}$ L'article publié dit : « On les menaçait du cachot».

${ }^{25}$ JACOB Max, Lettres à René Villard, op. cit., p. 101.

${ }^{26}$ GABORY Georges, préface, octobre 1921 dans JACOB Max, Le Cornet à dés, Paris, Librairie Stock, 1923, p. 9. Paragraphe initialement publié dans « Histoire d'une classe du lycée de Quimper », p. 86.

${ }^{27}$ Villard avait écrit : « Quand je le vois sous le portail moussu de l'église, en forme de conque marine, creusé de niches vidées de leur image de granit », ibid., p. 96.

${ }^{28}$ JACOB Max, Mon Chien noir, dans La Ligne de Cour, 5 novembre 1927 (JACOB Max, Lettres à René Villard, op. cit., p. 123 repris dans Poèmes de Morven le Gaélique, Paris, Gallimard, 1996, p. 57). ${ }^{29}$ Max Jacob publia des poèmes dans les premiers numéros de la revue fondée par Pierre Morhange (mars et novembre 1924) avant de rompre les liens avec le philosophe dont il contestait les choix politiques : « Nouveau Soviet signé Clarté, Surréalisme, Morhange etc.... ». Jacob « ne veut pas être avec les assassins» (JACOB Max, COCTEAU Jean, JACOB Max, Correspondance (1917-1944), correspondance annotée et présentée par Anne S. Kimball, Paris/Ripon, Paris Méditerranée/Écrits des Hautes-Terres, coll. Cachet Volant, 2000, p. 346). Concernant les rapports entre Jacob et le communisme voir HENRY Hélène, « Le voyage en Italie de Max Jacob et le fascisme italien » dans Antonio Rodriguez et Patricia Sustrac (dir.), Max Jacob face à l'histoire, actes de la journée d'étude du 6 février 2009, Université d'Orléans, Pau, Cahiers Max Jacob, nº 9, 2009, p. 53.

${ }^{30}$ À l'occasion de cette visite, Jacob dédicacera l'exemplaire de La Défense de Tartufe de Villard : « Tu as de la chance de posséder ce livre que je lirais volontiers si je l'avais. Il me ferait plus de bien à moi tiède catholique qu'il n'en ferait à ta ferveur. À René Villard ton ami Max Jacob, le 11 août 1927 ».

${ }^{31}$ Philippe Laignel Lavastine (1908-1999) est un jeune protégé du poète. Jacob l'avait rencontré en 1928 à l'Hôtel de la Madeleine dans l'entourage de Cocteau. Le jeune homme est le dédicataire de la réédition augmentée du Cabinet Noir, lettres avec commentaires (Librairie de France, Bibliothèque des Marges, 1922, rééd. NRF, 1928 ; Gallimard, coll. l'Imaginaire, 1968). Lavastine se consacrera à l'histoire des religions comparées et à la philosophie indienne. Séjournant en Inde, devenu sanskritiste, il est initié par plusieurs pandits à la science des Védas. De retour en France, il prodiguera un enseignement oral selon la tradition (MICHAEL Tara, Des Védas au Christianisme, hommage à Philippe Lavastine, Avignon, éd. Signatura, 2009).

${ }^{32}$ Florence Blumenthal, riche mécène américaine, initia la « Fondation pour la pensée et l'art français » après la première guerre afin d'attribuer des bourses à de jeunes artistes œuvrant pour le rayonnement de la pensée française. Jacques Rivière en fut le premier lauréat. Louis Guilloux obtiendra cette bourse en 1927 après la parution de La Maison du peuple.

${ }^{33}$ Pierre Gueguen (1889-1965) dont l'épouse Thérèse était effectivement très malade, était un poète proche des symbolistes, un prosateur et un critique d'art. Il rencontra Jacob en Bretagne en 1924.

Auteur de plusieurs ouvrages poétiques (Marées de Printemps, Paris, éd. Rieder, 1923 ; Arc-en-ciel sur la Domnonée, même éditeur, 1925), ce jeune poète n'a pas reçu de prix en 1927, date à laquelle son recueil Jeux Cosmiques avait commencé de paraître dans la revue Europe, avant d'être repris aux éditions Fourcade en 1929 (information communiquée par J.-F. Théry).

${ }^{34}$ Effectivement, Jacob ne tenait pas les prix littéraires en grande estime et ne reçut jamais les honneurs de prix prestigieux. En 1922, il confiait à Georges Gabory : « Je n’ai pas mérité de prix, réellement pas, m'étant fourvoyé dans le cubisme, alors que j'avais un talent d'ironiste désenchanté en naissant » (JACOB Max, Les Amitiés et les amours, lettres présentées par Didier Gompel, Paris, L'Arganier, t. I, 2005, p. 101). Cependant, il fut distingué en 1934 par le Comité de Noël du Poète. Mme George-Day, sa fondatrice, secrétaire générale de la SGDL, le pria de prononcer à cette occasion une allocution de 


\section{René Villard, biographe de Max Jacob}

circonstance que le poète transforma en un très important exposé esthétique dans le prolongement de sa préface du Cornet à dés (JACOB Max, Poèmes épars, le Cornet à dés II (une suite), choix et présentation par Claude Michel Cluny, coll. Orphée, Paris, La Différence, 1994).

${ }^{35}$ Le premier paragraphe seulement a été publié (JACOB Max, Lettres à René Villard, op. cit., p. 30, note 5). Repris dans le catalogue Max Jacob portrait d'artiste ( Paris, Somogy, 2004) il est cité, à tort, comme se situant à Quimper.

${ }^{36}$ Jacob cite également cette anecdote le 10 octobre 1927 dans une lettre à Maurice Sachs : «Grand fils/ Ce sont de grandes joies que tu me prépares et qui avaient été de grandes joies il y a quelques années mais je renonce tous les jours un peu plus : “Quand j'avais des dents je n'avais pas de bécasses, maintenant je n'ai plus de dents... " disait un vieil ami de ma famille à l'époque où on ne mettait pas de dentiers. Je n'ai pas de dentiers, cher Maurice » (lettre inédite, B. M. Orléans, ms 2549). ${ }^{37}$ Aucun des frères Jacob n'aura d'enfants, seule sa sœur cadette aura un fils (Robert Lévy, 19091980). Si les femmes ne sont pas absentes de l'univers jacobien, ses quelques aventures féminines connues (Cécile Aker, une jeune cousine...) resteront sans lendemains. À quel projet Villard, qui ne pouvait ignorer l'homosexualité de Jacob, fait-il allusion ? Cela ne peut être le projet trop lointain dont Jacob parle à Apollinaire en 1903 ou 1913 (BILLY André, Max Jacob, éd. Seghers, coll. Poète d'Aujourd'hui, 1969, p. 61), Jacob connaissait-il déjà, à cette époque, l'idée de Paul Poiret de le marier à Isadora Duncan (POIRET Paul, En habillant l'époque, Grasset, 1930, p. 91) ? Il n'est pas impossible que le mariage soit devenu une question de plaisanterie entre intimes. À Victor Moremans Jacob écrira : « J'ai failli me marier trois fois » (corr. inédite, lettre du 5 juillet 1932). Resté célibataire, Jacob - bien qu'il exhortait ses jeunes amis à trouver une épouse - est sans aucun doute plus concerné par la question de la paternité. Il a multiplié les fils spirituels notamment par le truchement de son abondante correspondance.

${ }^{38}$ Jacob écrit à Maurice Sachs le 30 avril : «Philippe a été un adorable enfant tant que je l'ai gâté ; quand il a senti que je voulais des progrès dans le sens homme et quand il a senti la férule, il a montré sa vraie nature qui est sensorielle, égoïste, incorrecte et hasardante. Comme il s'ennuyait à Ploaré, il a rejoint Liliane à Belle-Île et il est revenu reprendre les bagages ici. Depuis ce matin il est place Delaborde au 12bis et, je pense que son père m'expédiera un tout petit peu de ce que j'ai dépensé pour l'insouciant Philippe qui s'imagine que tout lui est dû, mais, pas "tout" pour "moi". Ainsi j’attendrai les événements » (lettre inédite, B. M. Orléans, op. cit.).

${ }^{39}$ Joseph Delteil n'a jamais écrit La vie de Saint Max Jacob. Les propos que rapporte Villard sont-ils une référence lointaine à l'article de Delteil Max Jacob et Dieu paru dans Le Disque Vert en 1923 ? Est-ce une confusion avec la biographie exaltée de de Richaud, Vie de Saint-Delteil, (Paris, éd. La Nouvelle Société d'Édition, 1928)?

${ }^{40}$ Concernant le voyage en Italie voir JACOB Max, Carnet Viaggio in Italia, testo originale a fronte, A cura di Adriano Marchetti, Marietti I Rombi, 2004, p. 16, édition bilingue. Concernant les motifs du voyage voir HENRY Hélène, « Le voyage en Italie de Max Jacob et le fascisme italien », op. cit. ${ }^{41}$ Kerhuel est un ancien élève du lycée de Quimper.

${ }^{42}$ Max Jacob avait l'art des dédicaces. La plus célèbre demeure celle de la réédition de Saint Matorel: « À Picasso, pour ce que je sais qu'il sait, pour ce qu'il sait que je sais » (NRF, 1936). Sur cet art typiquement jacobien, voir DURAND GUIZIOU Marie-Claire, « À propos d'une dédicace poétique à André Salmon », Journal de philologie de l'Université de Las Palmas, 2002-2003. 